\title{
Subclinical atherosclerosis and impaired bone health in patients with primary Sjogren's syndrome: prevalence, clinical and laboratory associations
}

Fotini Gravani ${ }^{1,2}$, loanna Papadaki ${ }^{1}$, Eleni Antypa ${ }^{3}$, Andrianos Nezos ${ }^{4}$, Kyriaki Masselou $^{5}$, Dimitrios loakeimidis ${ }^{1}$, Michael Koutsilieris ${ }^{4}$, Haralampos M Moutsopoulos ${ }^{2}$ and Clio P Mavragani ${ }^{2,4^{*}}$

\begin{abstract}
Introduction: To determine the prevalence and clinical/laboratory associations of subclinical atherosclerosis and impaired bone health in primary Sjogren's syndrome (SS).

Methods: 64 consecutive patients with primary SS, 77 with rheumatoid arthritis (RA) and 60 healthy controls (HC) of similar age and sex distribution were enrolled. Demographics, clinical/laboratory features, classical risk factors for atherosclerosis and osteoporosis (OP) were recorded. Intima-medial thickness scores (IMT) and carotid/femoral (C/F) plaque formation, as well as bone mineral density (BMD) and fractures were evaluated. Determinants of IMT/BMD levels and the presence of plaque were assessed by univariate and multivariate models. Serum levels of the Wnt signaling mediators Dickkopf-related protein 1(DKK1) and sclerostin were determined in primary SS patients and HC.

Results: Increased arterial wall thickening (IMT>0.90 mm) and impaired bone health (defined as OP or osteopenia), were detected in approximately two-thirds of primary SS and RA patients, with a mean IMT value being significantly increased compared to HC. The presence of primary SS emerged as an independent risk factor for arterial wall thickening when traditional risk factors for cardiovascular disease (CVD) including age, sex, hypertension, smoking (pack/years), LDL and HDL levels were taken into account in a multivariate model [adjusted OR 95\% (Cl): 2.8 (1.04-7.54)]. In primary SS, age was revealed as independent predictor of increased IMT scores; age and lymphopenia as well as increased urine pH as independent determinants of C/F plaque formation and OP/osteopenia, respectively. An independent association of OP/osteopenia with plaque formation was observed when independent predictors for both variables were considered, with low DKK1 levels being associated with both plaque formation and lower BMD levels.
\end{abstract}

Conclusions: Comorbidities such as subclinical atherosclerosis and impaired bone health occur frequently in primary SS, in association with disease related features and traditional risk factors. Wnt signaling mediators are potentially involved in the pathogenesis of both entities.

\section{Introduction}

Increased rates of subclinical atherosclerosis have been previously described in systemic autoimmune diseases, particularly in systemic lupus erythematosous (SLE) and rheumatoid arthritis (RA) [1-4]. However, traditional cardiovascular risk factors such as smoking, dyslipidemia, diabetes mellitus (DM), hypertension (HT) and increased

\footnotetext{
*Correspondence: kmauragan@med.uoa.gr

${ }^{2}$ Department of Pathophysiology, School of Medicine, University of Athens,

Athens, Greece

${ }^{4}$ Department of Physiology, School of Medicine, University of Athens, M.

Asias 75, Athens 11527, Greece

Full list of author information is available at the end of the article
}

body mass index (BMI), do not fully account for the high rates of subclinical atherosclerosis in these patients [5-7]. Additionally, osteopenia and osteoporosis (OP) rates, which have been found to be associated with surrogate markers of cardiovascular comorbidity in healthy populations [8], have been also shown to be increased in patients with SLE, RA and systemic sclerosis [9-11].

Sjogren's syndrome (SS) or autoimmune epithelitis is a slowly progressing autoimmune disease characterized by salivary and lacrimal gland dysfunction, resulting in oral and ocular dryness. The syndrome shares many common clinical features and serologic markers with other immunemediated autoimmune diseases, especially SLE $[12,13]$. In 
contrast to SLE, available data on comorbidities in SS are rather limited.

In the present study, we sought to determine the prevalence of subclinical atherosclerosis and impaired bone health (OP or osteopenia) in patients with primary SS and to explore whether they are associated with disease-related features and with traditional risk factors for cardiovascular disease (CVD) and OP. In addition, the Wingless-type (Wnt) signaling pathway, which has been implicated in the pathophysiology of both CVD and OP [14,15], was also explored.

\section{Methods}

\section{Study subjects}

In the present prospective cohort study, 64 consecutive patients with primary SS according to the AmericanEuropean Classification Criteria [16], 77 patients with RA fulfilling the American College of Rheumatology classification criteria for RA and 60 consecutive healthy individuals (of similar age and sex distribution to the patients with primary SS) were studied as disease and healthy controls $(\mathrm{HC})$, respectively. All patients were followed at the Department of Pathophysiology, School of Medicine, University of Athens and Department of Rheumatology, General Hospital of Athens G. Gennimatas. Exclusion criteria for all participants included pregnancy, age $<18$ years, renal dysfunction (serum creatinine levels $>3 \mathrm{mg} / \mathrm{dl}$, creatinine clearance $<30 \mathrm{ml} /$ minute). Serum was collected from patients and controls and stored at $-70^{\circ} \mathrm{C}$. The study has been approved by the Ethics Committee of Laikon General hospital and General Hospital of Athens G. Gennimatas. All patients provided written informed consent prior to their entry in the study.

\section{Clinical assessment}

Demographic data, clinical features, therapeutic regimens and classical risk factors for atherosclerosis and OP were recorded in all participating patients and controls. Demographic data included age, sex, and BMI. Clinical manifestations included the presence of subjective and objective oral and ocular dryness (documented by unstimulated salivary flow rates and Schirmer's test/Rose Bengal staining, respectively) [16]; dry cough; dyspareunia; fever; arthralgias; arthritis; carpal tunnel syndrome (documented by physical examination and nerve conduction studies); Raynaud's phenomenon; lymphadenopathy; splenomegaly; purpura; pulmonary involvement (small airway disease or interstitial lung disease documented by pulmonary function tests and high resolution computed tomography scans); pleuritis; pericarditis; renal involvement including interstitial nephritis (documented by urine-specific gravity $<1.010$ or $\mathrm{pH}>5.5$ on at least two consecutive measurements after fluid restriction) and glomerulonephritis documented by renal biopsy; liver involvement (documented by liver biopsy showing changes compatible with primary biliary cirrhosis in the setting of increased liver enzymes or anti-mitochondrial antibodies); peri-epithelial disease (defined as peribronchial, interstitial nephritis, autoimmune cholangitis); myositis (documented by muscle biopsy in the setting of increased aldolase or creatinophosphokinase); peripheral neuropathy (documented by nerve conduction studies in patients with clinical symptoms or signs suggestive of neuropathy); central nervous involvement; lymphoma (documented by biopsy) and other neoplastic diseases. Sjogren's syndrome disease activity index (ESSDAI) was also determined [17].

Classical risk factors for atherosclerosis and OP included the presence of family history of coronary disease (defined as a cardiovascular episode occurring below the age of 55 years in men and below 65 years in women in firstdegree relatives); family history of low-energy fracture; past medical history (PMH) of coronary heart disease, stroke, DM, HT, low-energy fractures, malabsorption, or thyroid function abnormalities; smoking history; alcohol consumption (units/weeks); age of menarche and menopause (for women) and total/current steroid use.

\section{Hematological, biochemical and immunological parameters} Full blood count results, Westergren erythrocyte sedimentation rate (ESR), C-reactive protein (CRP) levels (cut off value $5 \mathrm{mg} / \mathrm{l}$ ) and kidney, liver and thyroid function tests were recorded. Additionally, plasma levels of total cholesterol and high-density lipoprotein (HDL) cholesterol, low-density lipoprotein (LDL) cholesterol, triglyceride, homocysteine and fibrinogen levels were determined for all patients and controls, after overnight fasting. Serum and 24-hour urine calcium and phosphorus levels, intact parathyroid hormone $(\mathrm{PTH})(\mathrm{pg} / \mathrm{ml})$ and 25 (hydroxy) vitamin D $\left(25(\mathrm{OH}) \mathrm{D}_{3}\right)(\mathrm{ng} / \mathrm{ml})$ levels were also measured.

Immunological profile testing included antinuclear antibodies (ANA), antibodies to Ro (SSA) and La (SSB) antigens, complement levels, rheumatoid factor (RF) and second-generation anti-cyclic citrullinated peptide antibody (only in RA patients); hypergammaglobulinemia (total gamma globulins $>2 \mathrm{~g} / \mathrm{l}$ ) and cryoglobulins.

\section{Wnt signaling pathway}

To determine whether the Wnt signaling pathway is implicated in the concomitant presence of CVD and impaired bone health in patients with primary SS, serum levels of Dickkopf-related protein 1 (DKK1) and sclerostin were also determined in available sera from 59 patients with primary SS and $17 \mathrm{HC}$ by commercial ELISA, as per manufacturer instructions (R\&D Systems, Minneapolis, MN, USA). According to these results, SS patients were further subdivided in those characterized by low and high DKK1 and sclerostin levels. As a cut off value was 
considered the sum of the mean +2 SD of HC (for DKK1 $2403.6 \mathrm{pg} / \mathrm{ml}$ and sclerostin levels $212.2 \mathrm{pg} / \mathrm{ml}$ ).

\section{Assessment of subclinical atherosclerosis}

The presence of subclinical atherosclerosis was defined by the presence of plaque and/or arterial wall thickening (defined as intima media thickness (IMT) score $>0.90 \mathrm{~mm}$ in carotid and femoral arteries as determined by ultrasound (iU22, Philips, Royal Philips Electronics of the Netherlands)) [18]. Both the carotid (common carotid, bifurcation, and internal carotid) and the femoral (common femoral and superficial femoral) arteries were evaluated in each individual. The mean carotid artery IMT was defined as the average of 36 IMT readings (common, bifurcation, and internal carotid arteries, right and left side, far and near wall, with three sampling points per segment) and the mean femoral artery IMT was the average of twenty-four IMT readings (common and superficial femoral arteries, right and left side, far and near wall, with three sampling points per segment), as previously described [19]. Plaque formation was defined as a focal protrusion of more than $50 \%$ of the surrounding wall $[20,21]$. The radiologist (EA) in charge of the ultrasound scanning was unaware of the clinical diagnosis of the subjects under evaluation.

\section{Bone mineral density (BMD) measurements}

BMD measurements were performed in all patients and controls by dual-energy $x$-ray absorptiometry using the QDR4500 densitometer (Hologic, Bedford, MA, USA), and were cross calibrated. On the basis of the individual's age, BMD determination was performed either on the hip (total hip region) or the spine (anteroposterior lumbar spine, lumbar vertebrae L1 to L4), for ages above or below 65 years, respectively, according to previously published guidelines [22]. The presence of OP or osteopenia was defined according to the World Health Organization (WHO) classification system, as a T-score $<-2.5 \mathrm{SD}$ and $-1 \mathrm{SD}$, respectively, at either the lumbar spine or the hip [23].

\section{Statistical analysis}

Two-sided Fisher's exact/chi-square and Mann-Whitney tests were implemented to compare qualitative and quantitative characteristics, respectively, between patient groups and HC (Graph Pad Prism 5.00, GraphPad Software, San Diego, CA, USA). In order to explore the independent contribution of SS itself to the increased IMT levels, a multivariate model was constructed including age, sex, HT, smoking (pack/years), LDL and HDL. Determinants of arterial wall thickening (defined as IMT $>0.90 \mathrm{~mm}$ ) and plaque, and the presence of OP or osteopenia in the setting of SS and RA were assessed by univariate and multivariate models. Multivariate models included the traditional risk factors for CVD or OP/osteopenia, and disease-related features that were found to be significant in univariate analysis. In order to test whether the presence of plaque formation was independently associated with the presence of OP/osteopenia in primary SS and RA, another model was constructed including well-established common risk factors for both plaque formation and impaired bone health such as age, total steroid dose and history of smoking. A $P$ value $<0.05$ for univariate analyses and $<0.1$ for multivariate analyses, respectively, were considered statistically significant. Data were stored in the SPSS statistical package.

\section{Results}

\section{Demographics - traditional risk factors for CVD and OP}

Table S1 summarizes the demographic characteristics and prevalence of traditional risk factors for CVD and OP in patients with primary SS or RA, and in age- and sex-matched HC (see Additional file 1). As shown, the age distribution and sex ratio did not differ significantly among the three groups. Patients with primary SS had a mean age of $57.2 \pm 12.4$ years, a female-to-male ratio of 15 to 1 and disease duration of $8.4 \pm 7.0$ (mean \pm SD) years. Patients with RA had a mean age of $58.4 \pm$ 11.3 years, a female-to-male ratio of 14.6 to 1.0 and disease duration of $14.8 \pm 11.0$ years, which was significantly higher compared to the primary SS group. The corresponding values for $\mathrm{HC}$ were $56.4 \pm 7.8$ years and the female-to-male ratio was 14 to 1 .

In regard to traditional $\mathrm{CV}$ and $\mathrm{OP}$ risk factors, compared to the HC group, patients with primary SS had lower cholesterol levels and reduced rates of family history of CVD (mean \pm SD $194.6 \pm 34.2 \mathrm{mg} / \mathrm{dl}$ versus $211.8 \pm 32.2 \mathrm{mg} / \mathrm{dl}$; $P=0.01,14.2 \%$ versus $28.8 \% ; P=0.04$ respectively). Compared to the RA group, patients with primary SS had smaller disease duration ( $8.4 \pm 7.03$ versus $14.8 \pm 11.0$ years; $P=0.0004$ ), reduced current daily steroid dose intake $(1.3 \pm 2.2 \mathrm{mg}$ versus $4.8 \pm 4.7 \mathrm{mg} ; P=0.0001)$, lower serum triglyceride $(103.7 \pm 43.1 \mathrm{mg} / \mathrm{dl}$ versus $124.7 \pm 53.8 \mathrm{mg} / \mathrm{dl}$; $P=0.01)$ and CRP levels $(4.6 \pm 10.7 \mathrm{mg} / \mathrm{l}$ versus $14.8 \pm$ $27.9 \mathrm{mg} / \mathrm{l} ; P<0.0001)$ as well as higher $25(\mathrm{OH})$ vitamin $\mathrm{D}$ and phosphate levels $(21.3 \pm 11.4 \mathrm{ng} / \mathrm{ml}$ versus $17.5 \pm$ $7.1 \mathrm{ng} / \mathrm{ml} ; P=0.04$ and $3.3 \pm 0.5 \mathrm{mg} / \mathrm{dl}$ versus $3.1 \pm$ $0.5 \mathrm{mg} / \mathrm{dl} ; P=0.01$, respectively). On the other hand, RA patients demonstrated increased rates of DM (14.1\% versus $0 \%, P=0.002$ ), significantly elevated triglyceride levels $(124.7 \pm 53.8 \mathrm{mg} / \mathrm{dl}$ versus $104.8 \pm 53.2 \mathrm{mg} / \mathrm{dl} ; P=0.001)$, lower serum phosphate and 25(OH)D3 levels (3.1 \pm $0.5 \mathrm{mg} / \mathrm{dl}$ versus $3.4 \pm 0.4 \mathrm{mg} / \mathrm{dl} ; P=0.0008,17.5 \pm 7.1 \mathrm{ng} /$ $\mathrm{ml}$ versus $22.2 \pm 7.1 \mathrm{ng} / \mathrm{ml} ; P=0.0009)$ and increased CRP levels compared to $\mathrm{HC}(14.8 \pm 27.9 \mathrm{mg} / \mathrm{l}$ versus $2.7 \pm$ $2.3 \mathrm{mg} / \mathrm{l} ; P=0.0004$, respectively).

\section{Prevalence of comorbidities in patients with primary SS} or RA and in age- and sex-matched healthy individuals Increased IMT scores were detected in patients with primary SS and RA compared to HC (mean \pm SD $1.0 \pm 0.3$ 
and $1.0 \pm 0.4$ versus $0.9 \pm 0.2 ; P=0.03$ and $P=0.002$, respectively) (Figure 1, Table 1). Additionally, the rates of arterial wall thickening (defined as IMT $>0.90 \mathrm{~mm}$ ), carotid and femoral plaque formation, OP/osteopenia and low-energy fractures are displayed in Table 1 . The prevalence of arterial wall thickening was found to be increased in primary SS compared to the $\mathrm{HC}$ group (59.4\% versus $40.4 \%$, odds ratio (OR) 2.2 , $95 \%$ CI 1.02 , $4.55 ; P<0.05)$. Of interest, the presence of primary SS emerged as an independent risk factor for arterial wall thickening when traditional risk factors for CVD including age, sex, HT, smoking (pack/years), LDL and HDL levels were taken into account in a multivariate model (adjusted OR $2.8,95 \%$ CI 1.04, 7.54). Similar results were obtained for RA patients compared to HC (data not shown).

Additionally patients with RA (but not with SS) differed from $\mathrm{HC}$ in regard to plaque formation, OP/osteopenia and fractures $(84.4 \%$ versus $56.9, P<0.001,72.2 \%$ versus $50.9, P<0.05$ and $23.9 \%$ versus $8.5, P<0.05$, respectively). Compared to patients with primary SS, those with RA exhibited significantly higher rates of plaque formation and OP (84.4 versus 68.8, $P<0.05$ and $18.1 \%$ versus $7.8 \%$, $P<0.05$, respectively).

\section{Associations between IMT levels and traditional risk} factors for CVD and disease-related features in patients with primary SS

We next wished to identify predictors of arterial wall thickening in the setting of primary SS. In the primary SS group, a statistically significant association was observed between IMT levels and traditional CVD risk factors including age, BMI, HT, LDL and uric acid levels. Among disease-related

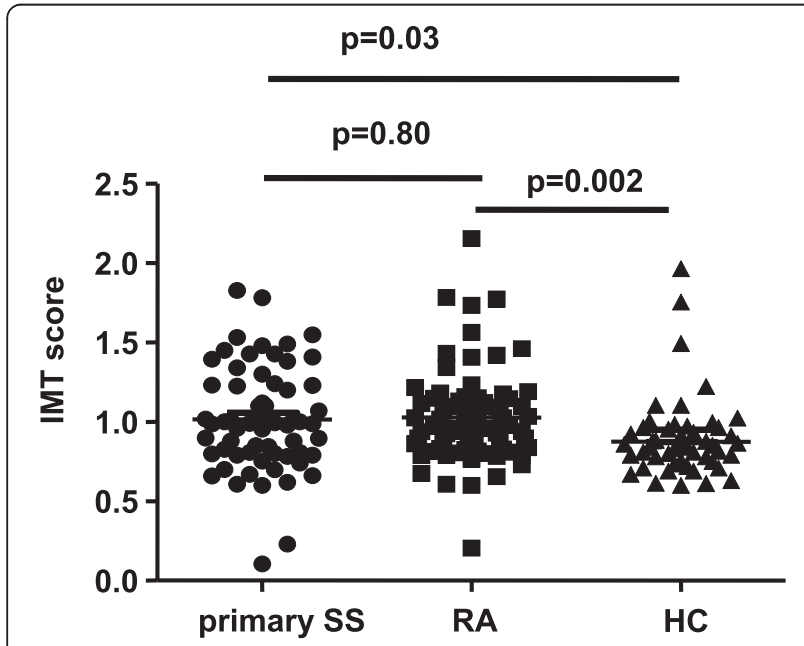

Figure 1 Intima media thickness (IMT) scores in patients with primary Sjogren's syndrome (SS) versus rheumatoid arthritis (RA) and healthy controls (HC). Increased IMT scores were detected in patients with primary SS or RA compared to $\mathrm{HC}$ (mean \pm SD $1.0 \pm 0.3$ and $1.0 \pm 0.4$ versus $0.9 \pm 0.2 ; P=0.03$ and $P=0.002$, respectively). features, the presence of peri-epithelial disease, increased levels of C3 and decreased urine specific gravity were also found to be significantly associated with increased IMT scores (see Table 2). Multivariate analysis revealed only age as independent predictor of arterial wall thickening in the setting of primary SS (OR 1.1, 95\% CI 1.1, 1.2; $P=0.002)$. No statistical significant associations were detected between total steroid dose and IMT score in primary SS ( $r=-0.07 ; P=0.57$, Spearman's rank correlation).

\section{Predictors of plaque formation in patients with primary SS}

The presence of carotid and/or femoral artery plaque in the univariate analysis was positively associated with age, the focus score on minor salivary gland biopsy and Raynaud's phenomenon, and was negatively associated with whole salivary flow rates and lymphocytic numbers (Table 3). Multivariate analysis confirmed age and absolute number of lymphocytes as independent predictors of plaque formation in the context of primary SS (OR 1.1, 95\% CI 1.0, 1.1 and OR 0.9, 95\% CI 0.9, 1.0, respectively).

\section{Determinants of OP and/or osteopenia in patients with primary SS}

In univariate analysis the classical risk factors of age and serum calcium levels were identified to be positively associated with the presence of OP and/or osteopenia in patients with primary SS. Additionally, patients with evidence of either osteopenia or OP exhibited lower rates of a positive family history of fracture, increased prevalence of arthralgia and higher mean values of urine $\mathrm{pH}$. In multivariate analysis serum calcium and increased urine $\mathrm{pH}$ emerged as independent predictors of OP or osteopenia among patients with primary SS (OR 3.2, 95\% CI 1.01, 10.4) and OR 4.5, 95\% CI 1.004, 20.4, respectively) (Table 4).

Further analysis of the prevalence of OP and/or osteopenia was subsequently carried out by subdividing the patients with primary SS according to the urinary $\mathrm{pH}$ levels (cut off 5.5). According to this subgroup analysis, the patients with primary SS who had urinary $\mathrm{pH}>5.5$ at the first visit exhibited significantly increased rates of OP or osteopenia compared to those with urinary $\mathrm{pH}<5.5$ (94.3\% versus $44.4 \%, P=0.002$ ) (Additional file 2: Figure $\mathrm{S} 1$ ).

In a subset of 57 patients with primary SS, urine levels of type 1 cross-linked c-telopeptide (CTX) were determined; no statistical significant associations were detected between CTX levels, BMD levels, total steroid dose and SS-activity index as analyzed by Spearman's rank correlation (data not shown).

\section{Independent association of impaired bone health with} atherosclerotic plaque formation in patients with primary SS Given the previously reported associations between alterations in bone mass with atherosclerotic risk in healthy 
Table 1 Prevalence of comorbidities in patients with primary SS or RA and in age- and sex-matched healthy individuals

\begin{tabular}{|c|c|c|c|c|c|c|}
\hline & SS (n=64) & $\mathrm{RA}(\mathrm{n}=77)$ & $H C(n=60)$ & $P$-value* & $P$-value** & $P$-value ${ }^{* * *}$ \\
\hline Arterial wall thickening (IMT >0.90 mm), \% & 59.4 & $63.8^{a}$ & 40.4 & ns & $<0.05$ & $<0.01$ \\
\hline IMT levels, mean \pm SD & $1.0 \pm 0.3$ & $1.0 \pm 0.4^{\mathrm{a}}$ & $0.9 \pm 0.2$ & ns & $<0.05$ & $<0.01$ \\
\hline Presence of plaque, $\%$ & 68.8 & 84.4 & 56.9 & $<0.05$ & ns & $<0.001$ \\
\hline Osteopenia, \% & 51.6 & $54.2^{\mathrm{a}}$ & 36.8 & ns & ns & ns \\
\hline Osteoporosis, \% & 7.8 & $18.1^{\mathrm{a}}$ & 14.0 & ns & ns & ns \\
\hline Osteoporosis/osteopenia, \% & 59.4 & $72.2^{\mathrm{a}}$ & 50.9 & ns & ns & $<0.05$ \\
\hline Fractures, \% & 12.5 & $23.9^{\mathrm{a}}$ & 8.5 & ns & ns & $<0.05$ \\
\hline
\end{tabular}

*Primary Sjogren's syndrome (SS) versus rheumatoid arthritis (RA); ${ }^{* *}$ primary SS versus healthy controls (HC); ${ }^{* * *}$ RA versus HC; ${ }^{a}$ analysis based on 72 patients with RA. IMT, intima media thickness; ns, not significant.

populations, we sought to explore similar associations in an autoimmune population, such as patients with primary SS $[24,25]$. In fact, the presence of either OP or osteopenia in our SS study group was found to be strongly associated with the presence of plaque, as detected by carotid and femoral ultrasound (OR 4.0, 95\% CI 1.3, 12.2; $P=0.01$ ) with $80 \%$ of patients with plaque having evidence of impaired bone health versus $20 \%$ of those with no plaque (Figure 2A). This association remained significant when classical predictors of both clinical entities, such as age, total steroid dose and smoking, were included in the multivariate model.

In order to investigate whether the Wnt signaling system was involved in the concomitant occurrence of both atherosclerotic and osteoporotic disease in our SS patients, DKK1 and sclerostin levels were also determined by commercial ELISA. A positive correlation between sclerostin and DKK1 levels with neck BMD levels was detected $(r=0.42 ; P=0.02$ and $r=0.41 ; P=0.01$, respectively). However, no association was found between sclerostin levels and the presence of atherosclerotic plaque formation (data not shown). On the other hand, patients with primary SS characterized by low DKK1 levels compared to those with high DKK1 levels had reduced bone mass levels $\left(0.7 \pm 0.1\right.$ versus $\left.0.9 \pm 0.1 \mathrm{mg} / \mathrm{cm}^{2}\right)$ and increased rates of plaque formation (81.8\% versus $53.3 \%$ ), implying Wnt signaling as a potential contributor for the concomitant presence of both comorbidities in a subgroup of patients with primary SS (Figure $2 \mathrm{~B}$ and $\mathrm{C}$ ).

\section{Associations between IMT levels and plaque formation with traditional risk factors for CVD and disease-related features in RA patients}

In univariate analysis, arterial wall thickening among RA patients was identified to be positively associated with age, BMI, triglyceride levels, number of tender joints and blood urea nitrogen (BUN) levels, and negatively associated with cyclosporine treatment, CRP levels and number of platelets $/ \mathrm{mm}^{3}$. Age, BMI, the number of tender joints and cyclosporine use were identified as independent predictors of arterial wall thickening in the setting of RA in multivariate analysis (see Additional file 1: Table S2). No statistically significant associations were detected between total steroid dose and IMT score in the RA population ( $r=0.20 ; P=0.89$, Spearman's rank correlation).

RA patients with carotid and/or femoral artery plaque were older, with higher BMI, higher cumulative steroid doses and BUN levels and increased rates of HT compared to those without plaque formation. Paradoxically, plaque formation was negatively associated with history of smoking among RA patients in univariate analysis (see Additional file 1: Table S3). Multivariate analysis confirmed age and total steroid dose as independent predictors of plaque formation in the context of RA.

\section{Determinants of OP and/or osteopenia in RA patients}

In both univariate and multivariate analysis, increased age, $\mathrm{PMH}$ of fracture and increased $\mathrm{PTH}$ were identified to be positively associated with the presence of OP and/or osteopenia in RA patients (see Additional file 1: Table S4).

\section{Discussion}

In the present study we wished first to determine the prevalence of comorbidities such as subclinical atherosclerosis and impaired bone health in a cohort of patients with primary SS compared to healthy and RA individuals with a similar age/sex distribution, and second, to explore potential associations with disease-related features and traditional risk factors for CVD and OP. We have shown that the presence of primary SS was as an independent risk factor for arterial wall thickening, when traditional risk factors for CVD including age, sex, HT, smoking (pack/years), LDL and HDL levels were taken into account in a multivariate model. While the carotid/femoral IMT levels were found to be significantly higher in primary SS and RA patients versus $\mathrm{HC}$, only RA patients demonstrated increased rates of plaque formation compared to both SS patients and $\mathrm{HC}$. The higher proportion of DM and the increased triglyceride and CRP levels, together 
Table 2 Traditional and disease-related predictors of arterial wall thickening in patients with primary SS

\begin{tabular}{|c|c|c|c|c|c|}
\hline & \multirow{2}{*}{$\begin{array}{l}\text { Univariate analysis } \\
\text { IMT >0.90 }(n=38)\end{array}$} & \multirow[b]{2}{*}{ IMT $\leq 0.90(n=26)$} & \multirow[b]{2}{*}{$P$-value } & \multicolumn{2}{|l|}{ Multivariate analysis } \\
\hline & & & & Odds ratio $(95 \% \mathrm{Cl})$ & $P$-value \\
\hline \multicolumn{6}{|l|}{ Traditional risk factors } \\
\hline Age, years & $62.5 \pm 9.2$ & $49.8 \pm 12.6$ & $<0.001$ & $1.1(1.1,1.2)$ & 0.002 \\
\hline Female, \% & 92.1 & 96.2 & ns & & \\
\hline Past medical history of CVD, \% & 7.9 & 3.8 & ns & & \\
\hline Family history of CVD, \% & 13.2 & 15.4 & ns & & \\
\hline Smoking, packs/year & $10.5 \pm 20.7$ & $3.6 \pm 7.4$ & ns & & \\
\hline Body mass index & $29.0 \pm 5.7$ & $25.0 \pm 4.4$ & 0.02 & & \\
\hline Diabetes, \% & 5.3 & 7.7 & ns & & \\
\hline Hypertension, \% & 47.4 & 19.2 & 0.04 & & \\
\hline Cholesterol levels, mg/dl & $202.2 \pm 35.2$ & $183.8 \pm 30.0$ & ns & & \\
\hline High-density lipoprotein, mg/dl & $57.0 \pm 13.9$ & $53.5 \pm 15.5$ & ns & & \\
\hline Low-density lipoprotein, mg/dl & $126.8 \pm 32.2$ & $111.2 \pm 25.0$ & 0.03 & & \\
\hline Triglycerides, mg/dl & $111.5 \pm 44.7$ & $92.5 \pm 38.8$ & ns & & \\
\hline Homocysteine levels, $\mu \mathrm{mol} / \mathrm{l}$ & $14.6 \pm 4.6$ & $14.2 \pm 6.0$ & ns & & \\
\hline Uric acid, mg/dl & $4.4 \pm 1.1$ & $3.6 \pm 1.2$ & 0.006 & & \\
\hline Current steroid dose, mg & $1.3 \pm 2.3$ & $1.1 \pm 2.1$ & ns & & \\
\hline Total steroid dose, $\mathrm{g}$ & $7.8 \pm 17$ & $6.6 \pm 11.3$ & ns & & \\
\hline Current TSH levels, $\mu$ IU/dl & $1.5 \pm 1.4$ & $1.8 \pm 1.3$ & ns & & \\
\hline C-reactive protein, mg/l & $3.1 \pm 3.2$ & $6.7 \pm 16.0$ & ns & & \\
\hline Fibrinogen, mg/dl & $416.6 \pm 108.2$ & $395.6 \pm 128.1$ & ns & & \\
\hline 25-hydroxy vitamin D3, ng/ml & $22.3 \pm 13.4$ & $19.8 \pm 7.6$ & ns & & \\
\hline \multicolumn{6}{|l|}{ Disease-related features } \\
\hline Disease duration, years & $8.7 \pm 7.4$ & $8.0 \pm 6.6$ & ns & & \\
\hline SS disease activity index & $1.8 \pm 1.5$ & $1.8 \pm 1.7$ & ns & & \\
\hline Focus score, number of foci/ $4 \mathrm{~mm}^{2}$ & $2.6 \pm 2.2$ & $1.6 \pm 1.4$ & ns & & \\
\hline Whole salivary flow, $\mathrm{ml} / 15$ minutes & $2.0 \pm 2.8$ & $2.6 \pm 3.1$ & ns & & \\
\hline Peri-epithelial disease, \% & 31.6 & 3.8 & 0.007 & & \\
\hline Arthritis, \% & 23.6 & 46.1 & ns & & \\
\hline Arthralgias, \% & 65.7 & 76.9 & ns & & \\
\hline Dry mouth, \% & 84.2 & 76.9 & ns & & \\
\hline Dry eyes, \% & 86.8 & 88.5 & ns & & \\
\hline Salivary gland enlargement, $\%$ & 21.1 & 15.3 & ns & & \\
\hline Raynaud's phenomenon, \% & 28.9 & 23.1 & ns & & \\
\hline Lymphadenopathy, \% & 18.4 & 19.2 & ns & & \\
\hline Splenomegaly, \% & 2.6 & 3.8 & ns & & \\
\hline Palpable purpura, $\%$ & 10.5 & 3.8 & ns & & \\
\hline Monoclonal gammopathy (\%) & 10.5 & 3.8 & ns & & \\
\hline Cryoglobulins (\%) & 2.6 & 0 & ns & & \\
\hline Lymphoma (\%) & 18.4 & 7.6 & ns & & \\
\hline Antinuclear antibodies $>1 / 160, \%$ & 89.4 & 96.1 & ns & & \\
\hline Anti-Ro/SSA, \% & 76.3 & 73.1 & ns & & \\
\hline Anti-La/SSB, \% & 42.1 & 57.7 & ns & & \\
\hline
\end{tabular}


Table 2 Traditional and disease-related predictors of arterial wall thickening in patients with primary SS (Continued)

\begin{tabular}{|c|c|c|c|}
\hline Rheumatoid factor titers, IU/ml & $61.0 \pm 104$ & $58.3 \pm 84.0$ & ns \\
\hline Complement 3, mg/ml & $1.2 \pm 0.2$ & $1.1 \pm 0.2$ & 0.002 \\
\hline Complement 4, mg/ml & $0.2 \pm 0.1$ & $0.2 \pm 0.1$ & ns \\
\hline Absolute number of $\mathrm{WBC} / \mathrm{mm}^{3}$ & $5,647 \pm 1903$ & $5,727 \pm 1785$ & ns \\
\hline Absolute number of lymphocytes $/ \mathrm{mm}^{3}$ & $1,518 \pm 463$ & $1,645 \pm 756$ & ns \\
\hline Erythrocyte sedimentation rate & $34 \pm 21$ & $31 \pm 22$ & ns \\
\hline Y-globulins, \% & $18.5 \pm 5.2$ & $18.6 \pm 3.4$ & ns \\
\hline Urine specific gravity & $1,013 \pm 6$ & $1,018 \pm 8.0$ & 0.007 \\
\hline Urine $\mathrm{pH}$ & $6.1 \pm 0.7$ & $6.1 \pm 0.6$ & ns \\
\hline
\end{tabular}

IMT, intima media thickness; SS, Sjogren's syndrome; IMT, Intima media thickness; CVD, cardiovascular disease; TSH, thyroid stimulating hormone; WBC, white blood cells; ns, not significant.

with the lower vitamin D levels observed in the RA cohort could account for the pronounced atherosclerotic risk in these patients, as previously reported [26-28].

We next aimed to explore whether disease-related and/ or traditional risk factors could account for the increased rates of comorbidities among patients with primary SS. Advanced age, higher hypertension rates and BMI, increased LDL and uric acid levels and the presence of periepithelial disease were found to be associated with arterial wall thickening, while advanced age, higher focus score on minor salivary gland biopsy and reduced salivary flow rates, the presence of Raynaud's syndrome and reduced lymphocytic numbers were predictors of plaque formation. In multivariate models, age was an independent predictor of both arterial wall thickening and plaque formation, and lymphopenia was an independent risk factor for plaque formation. Although increased prevalence of DM [29], hypertriglyceridemia [29,30], HT [30] and dyslipidemia [31,32] were previously reported to be higher among SS populations, we have not observed significant differences between patients with primary SS and $\mathrm{HC}$, except for lower rates of family history of CVD and cholesterol levels in the primary SS group. The latter observation was also supported by Lodde et al. [33].

Although the increased atherosclerotic risk is wellestablished in patients with SLE [34], RA [35] and systemic sclerosis [36], a limited body of evidence is available on primary SS syndrome. Vaudo et al., observed increased carotid and femoral IMT scores in white women with primary SS compared to age- and sex-matched $\mathrm{HC}$ in association with leucopenia and the presence of anti-SSA antibodies [18,19]. Although no association with anti-Ro/SSA antibodies and IMT values was detected in our cohort, we also revealed lymphopenia as a significant predictor of plaque formation in our patient group. Additionally, endothelial dysfunction identified by abnormal ankle brachial index (ABI), lower endotheliumdependent flow-mediated vasodilatation (FMV), increased asymmetric dimethylarginine (ADMA) and higher pulse wave velocity (PWV) values were recently demonstrated [37-39]. At the time of preparation of the current manuscript, data from a large multicenter Italian study demonstrated an increased risk of cerebrovascular events and myocardial infarction among primary SS populations [32]. On the other hand, impaired smooth-muscle relaxation was present in patients with primary SS independent of endothelial contribution, and particularly in those characterized by leucopenia, RF, anti-SSB antibodies and articular involvement [38].

The mechanisms for the observed atherosclerotic risk in SS have not yet been elucidated. Data derived mainly from lupus studies suggest that $\mathrm{T}$ and $\mathrm{B}$ cell dysregulation heightens production of cytokines such as inteleukin-17 (IL-17), interferon $\gamma$ (IFN $\gamma$ ), and more recently interferon $\alpha$ (IFN $\alpha)$, a central cytokine in both lupus and SS pathogenesis, which have been all considered to contribute to the increased cardiovascular risk of these patients. Particularly, impairment of endothelial repair through accelerated apoptosis of endothelial progenitor cells, together with enhancement of foam cell formation in the atherosclerotic plaque, have been proposed to account for the atherogenic potential of IFN $\alpha$ [40].

Age, BMI and clinical variables of disease activity as evidenced by increased number of tender joints were independent predictors of increased IMT scores in the RA group, whereas cyclosporine use had a protective role against arterial wall thickening among these patients, in line with previous observations in SLE patients, in which use of cyclosporine A protected against increased carotid IMT, leading to a decreased risk of arteriosclerosis [41]. Plaque formation was independently associated with age and total steroid dose, in line with previous observations [42].

Although an increased risk of OP and fracture rates in autoimmune diseases such as SLE [9], RA [43] and systemic sclerosis [11] is well-documented, no information is available on the occurrence of OP/osteopenia or fracture rates in patients with primary SS. Patients with 
Table 3 Traditional and disease-related predictors of plaque formation in patients with primary SS

\begin{tabular}{|c|c|c|c|c|c|}
\hline & \multicolumn{3}{|c|}{ Univariate analysis } & \multicolumn{2}{|l|}{ Multivariate analysis } \\
\hline & Plaque $(n=44)$ & No plaque $(n=20)$ & $P$-value & Odds ratio $(95 \% \mathrm{Cl})$ & $P$-value \\
\hline \multicolumn{6}{|l|}{ Traditional risk factors } \\
\hline Age, years & $60.1 \pm 9.1$ & $51.0 \pm 16.2$ & 0.006 & $1.1(1.0,1.1)$ & 0.09 \\
\hline Female, \% & 93.2 & 95 & ns & & \\
\hline Past medical history of CVD, \% & 6.8 & 5 & ns & & \\
\hline Family history of CVD, \% & 18.2 & 5 & ns & & \\
\hline Smoking, packs/year & $9.1 \pm 19.3$ & $4.7 \pm 9.3$ & ns & & \\
\hline Body mass index & $27.6 \pm 5.6$ & $26.8 \pm 5.5$ & ns & & \\
\hline Diabetes, \% & 9.1 & 0 & ns & & \\
\hline Hypertension, \% & 38.6 & 30.0 & ns & & \\
\hline Cholesterol levels, mg/dl & $194.4 \pm 33.9$ & $194.9 \pm 35.7$ & ns & & \\
\hline High-density lipoprotein, mg/dl & $56.6 \pm 15.1$ & $51.2 \pm 12.8$ & ns & & \\
\hline Low-density lipoprotein, mg/dl & $116.1 \pm 30.6$ & $130.2 \pm 27.7$ & ns & & \\
\hline Triglycerides, mg/dl & $107.8 \pm 43.9$ & $93.9 \pm 40.8$ & ns & & \\
\hline Homocysteine levels, $\mu \mathrm{mol} / \mathrm{l}$ & $15.3 \pm 5.5$ & $12.4 \pm 3.8$ & ns & & \\
\hline Uric acid, mg/dl & $4.1 \pm 1.3$ & $4.0 \pm 0.9$ & ns & & \\
\hline Current steroid dose, mg & $1.3 \pm 2.2$ & $1.1 \pm 2.2$ & ns & & \\
\hline Total steroid dose, $\mathrm{g}$ & $9.3 \pm 17.5$ & $3.7 \pm 6.3$ & ns & & \\
\hline Current TSH levels, $\mu$ IU/dl & $1.6 \pm 1.5$ & $1.6 \pm 1.2$ & ns & & \\
\hline C-reactive protein, mg/l & $3.2 \pm 5.8$ & $7.6 \pm 17.0$ & ns & & \\
\hline Fibrinogen, mg/dl & $410.0 \pm 99.7$ & $402.8 \pm 151.2$ & ns & & \\
\hline 25-hydroxy vitamin D3, ng/ml & $21.1 \pm 12.9$ & $21.7 \pm 7.0$ & ns & & \\
\hline \multicolumn{6}{|l|}{ Disease-related features } \\
\hline Disease duration, years & $8.9 \pm 7.3$ & $7.3 \pm 6.6$ & ns & & \\
\hline SS disease activity index & $1.7 \pm 1.5$ & $2.1 \pm 1.8$ & ns & & \\
\hline Focus score, number of foci/ $4 \mathrm{~mm}^{2}$ & $2.7 \pm 1.9$ & $1.2 \pm 1.4$ & 0.04 & & \\
\hline Whole salivary flow, $\mathrm{ml} / 15$ minutes & $1.9 \pm 2.8$ & $3.0 \pm 3.2$ & 0.04 & & \\
\hline Peri-epithelial disease, \% & 22.7 & 15.0 & ns & & \\
\hline Arthritis, \% & 29.5 & 40.0 & ns & & \\
\hline Arthralgias, \% & 68.2 & 75.0 & ns & & \\
\hline Dry mouth, \% & 84.1 & 75.0 & ns & & \\
\hline Dry eyes, \% & 90.9 & 80.0 & ns & & \\
\hline Salivary gland enlargement, $\%$ & 11.4 & 20 & ns & & \\
\hline Raynaud's phenomenon, \% & 34.1 & 10.0 & 0.04 & & \\
\hline Lymphadenopathy, \% & 18.2 & 20.0 & ns & & \\
\hline Splenomegaly, \% & 2.3 & 5 & ns & & \\
\hline Palpable purpura, $\%$ & 9.1 & 5 & ns & & \\
\hline Cryoglobulins (\%) & 2.3 & 0 & ns & & \\
\hline Lymphoma (\%) & 13.6 & 15.0 & ns & & \\
\hline Antinuclear antibodies >1/160, \% & 86.4 & 95 & ns & & \\
\hline Anti-Ro/SSA, \% & 72.7 & 75 & ns & & \\
\hline Anti-La/SSB, \% & 47.7 & 50.0 & ns & & \\
\hline Rheumatoid factor titers, IU/ml & $53.7 \pm 88.7$ & $72.5 \pm 108.6$ & ns & & \\
\hline
\end{tabular}


Table 3 Traditional and disease-related predictors of plaque formation in patients with primary SS (Continued)

\begin{tabular}{|c|c|c|c|c|c|}
\hline Complement 3, mg/ml & $1.1 \pm 0.2$ & $1.2 \pm 0.3$ & ns & & \\
\hline Complement 4, mg/ml & $0.2 \pm 0.1$ & $0.2 \pm 0.1$ & ns & & \\
\hline Absolute number of $\mathrm{WBC} / \mathrm{mm}^{3}$ & $5,533 \pm 1968$ & $6,006 \pm 1516$ & ns & & \\
\hline Absolute number of lymphocytes $/ \mathrm{mm}^{3}$ & $1,411 \pm 476$ & $1,924 \pm 705$ & 0.001 & $0.9(0.9,1.0)$ & 0.02 \\
\hline Erythrocyte sedimentation rate & $33 \pm 21$ & $31 \pm 21$ & ns & & \\
\hline Y-globulins, \% & $18.3 \pm 5.1$ & $19.0 \pm 2.7$ & ns & & \\
\hline Urine specific gravity & $1,014 \pm 6$ & $1,016 \pm 9$ & ns & & \\
\hline Urine $\mathrm{pH}$ & $6.1 \pm 0.7$ & $6.1 \pm 0.5$ & ns & & \\
\hline
\end{tabular}

IMT, intima media thickness; SS, Sjogren's syndrome; IMT, Intima media thickness; CVD, cardiovascular disease; TSH, thyroid stimulating hormone; WBC, white blood cells; ns, not significant.

primary SS share a number of clinical and serological features which theoretically predispose to low bone mineralization; these include low vitamin D levels, hypercalciuria related to underlying interstitial nephritis, steroid use for systemic involvement, and coexistence with other organ-specific autoimmune disorders known to increase OP risk, such as primary biliary cirrhosis and celiac disease [44-46]. In our hands, the rate of positive antimitochondrial as well as antigliandin and antitransglutaminase antibodies, respectively, was low, and therefore firm conclusions cannot be drawn (data not shown). In regard to $\mathrm{OP} /$ osteopenia and fracture rates and in

Table 4 Determinants of osteoporosis (OP) or osteopenia in patients with primary SS

\begin{tabular}{|c|c|c|c|c|c|}
\hline & \multicolumn{3}{|l|}{ Univariate analysis } & \multicolumn{2}{|l|}{ Multivariate analysis } \\
\hline & $\begin{array}{l}\text { Presence of osteoporosis } \\
\text { or osteopenia }(n=38)\end{array}$ & $\begin{array}{l}\text { Absence of osteoporosis } \\
\text { or osteopenia }(n=24)\end{array}$ & $P$-value & Odds ratio $95 \%(\mathrm{Cl})$ & $P$-value \\
\hline \multicolumn{6}{|l|}{ Classical risk factors for osteoporosis } \\
\hline Age, years & 59. $9 \pm 10.1$ & $52.8 \pm 14.6$ & 0.03 & & \\
\hline Family history of fracture, $\%$ & 5.4 & 27.2 & 0.02 & & \\
\hline Past medical history of fracture, $\%$ & 15 & 8.3 & ns & & \\
\hline Body mass index & $26.9 \pm 4.7$ & $28.2 \pm 6.8$ & ns & & \\
\hline Age of menarche, years & $13.4 \pm 1.7$ & $13.1 \pm 2.4$ & ns & & \\
\hline Age of menopause, years & $49.4 \pm 4.1$ & $50.6 \pm 6.3$ & ns & & \\
\hline Calcium, mg/dl & $9.7 \pm 0.5$ & $9.3 \pm 0.6$ & 0.02 & $3.2(1.01,10.4)$ & $<0.05$ \\
\hline Phosphate, mg/dl & $3.4 \pm 0.5$ & $3.2 \pm 0.6$ & ns & & \\
\hline Calcium urine/24 h, g & $0.1 \pm 0.1$ & $0.1 \pm 0.2$ & ns & & \\
\hline Phosphate urine/24 h, g & $0.5 \pm 0.2$ & $0.6 \pm 0.2$ & ns & & \\
\hline 25-hydroxy-vitamin D3, ng/ml & $21.2 \pm 12.9$ & $21.4 \pm 8.1$ & ns & & \\
\hline Parathyroid hormone, pg/ml & $50.2 \pm 22.6$ & $41.6 \pm 16.6$ & ns & & \\
\hline Current thyroid stimulating hormone levels ( $\mu \mathrm{IU} / \mathrm{dl}$ ) & $1.34 \pm 2.1$ & $1.3 \pm 1.5$ & ns & & \\
\hline Total steroid dose, g & $9.8 \pm 17.8$ & $3.2 \pm 0.5$ & ns & & \\
\hline Current steroid dose, mg/day & $1.6 \pm 2.4$ & $0.5 \pm 1.4$ & ns & & \\
\hline \multicolumn{6}{|l|}{ Disease-related features } \\
\hline Sjogren's syndrome activity index & $1.85 \pm 1.49$ & $1.74 \pm 1.8$ & ns & & \\
\hline Disease duration, years & $9.1 \pm 7.4$ & $7.3 \pm 6.3$ & ns & & \\
\hline Focus score, number of lymphocytic foci/4 mm² & $1.9 \pm 1.8$ & $2.4 \pm 1.9$ & ns & & \\
\hline Whole salivary flow, $\mathrm{ml} / 15$ minutes & $2.0 \pm 2.9$ & $2.6 \pm 3.1$ & ns & & \\
\hline Urine $\mathrm{pH}$ & $6.3 \pm 7.4$ & $5.8 \pm 0.4$ & 0.03 & $4.5(1.004,20.4)$ & $<0.05$ \\
\hline Urine specific gravity & $1,014 \pm 6.9$ & $1,015 \pm 7$ & ns & & \\
\hline Arthralgias, \% & 80 & 54.2 & 0.03 & & \\
\hline Peri-epithelial disease, \% & 27.5 & 8.3 & ns & & \\
\hline
\end{tabular}

ns, not significant. 


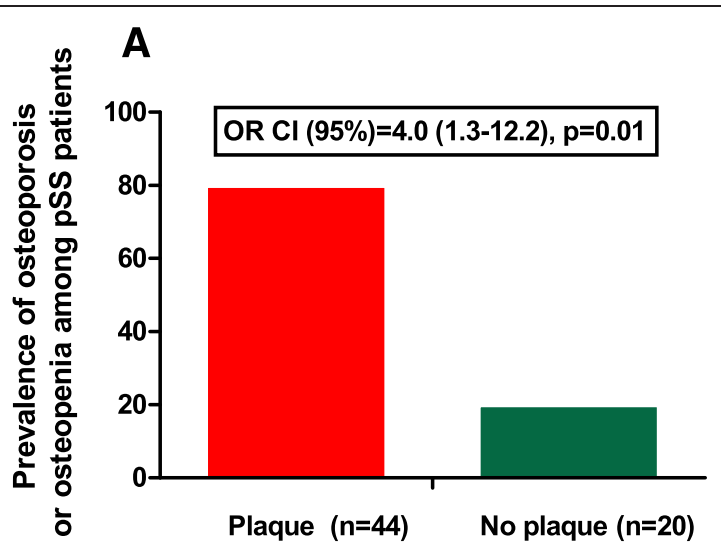

B
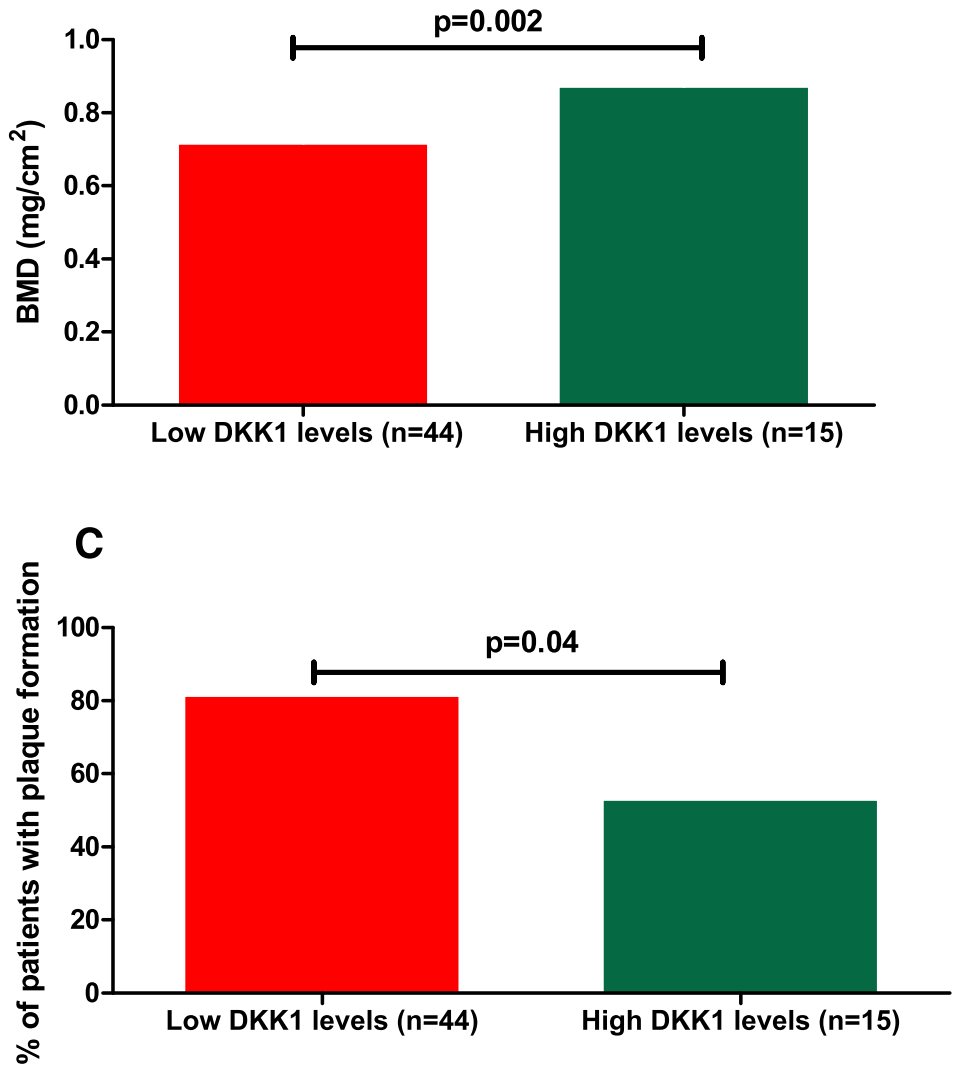

Figure 2 Independent association of osteopenia/osteoporosis (OP) with atherosclerotic plaque formation in patients with primary Sjogren's syndrome (SS). (A) Association of osteopenia/OP with plaque formation in SS (odds ratio (OR) 4.0, 95\% Cl 1.3, 12.2, P = 0.01). This association remained significant when potential confounders for both clinical entities, such as age, total steroid dose and smoking, were taken in consideration. (B) Patients with primary SS characterized by low Dickkopf-related protein 1 (DKK1) levels had reduced bone mass levels compared to those with high DKK1 levels $\left(0.7 \pm 0.1\right.$ versus $\left.0.9 \pm 0.1 \mathrm{mg} / \mathrm{cm}^{2}, P=0.002\right)$. (C) Similarly, patients with primary SS with low DKK1 levels had increased rates of plaque formation (81.8\% versus 53.3\%). The sum of the mean +2 SD of healthy controls (HC) (2403.6 pg/ml) was considered as the cut off value for low and high DKK1 levels.

contrast with RA patients, no statistically significant differences were observed between primary SS group as a whole and the $\mathrm{HC}$ group. It was surprising that patients with primary SS experienced paradoxically higher rates of fractures compared to $\mathrm{HC}$, despite the lower rates of osteopenia. Given that the differences noted were not statistically significant, we presume that this was a chance finding.

When patients with primary SS were stratified according to urine $\mathrm{pH}$, which was an independent predictor of $\mathrm{OP}$ and or/osteopenia in the multivariate analysis, significantly increased prevalence of OP/osteopenia was 
observed compared to the controls. Although vitamin D and serum phosphate levels were decreased in RA patients compared to $\mathrm{HC}$, which might contribute to the increased rates of $\mathrm{OP}$ and fracture risk in this population, they were within normal limits within our SS cohort. Additionally, mean current steroid dose and CRP levels as a marker of systemic inflammation, which was previously implicated in osteoporotic risk among RA patients [47], were increased in our RA cohort, in which independent predictors of OP/osteopenia included $\mathrm{PMH}$ of fracture, increased serum levels of PTH and advanced age, as previously reported [48].

A growing body of evidence over the last decade supports the association between bone mass reduction and atherosclerotic risk in healthy populations $[49,50]$. In the present study, we also observed the concomitant occurrence of decreased bone mass and plaque formation in our autoimmune SS group, when potential confounders for both clinical entities, such as age, total steroid dose and smoking were considered. Such an association was not detected in our RA cohort, possibly related to the confounding effects of concomitant medications.

To further explore the underlying linking mechanisms between impaired bone health and atherosclerotic risk in primary SS, inhibitors of the Wnt signaling pathway were measured, including sclerostin and DKK1, which were previously potentially implicated in the pathogenesis of arterial calcification and/or inhibition of bone formation [51-53]. In accordance with previously reported findings in diabetic [52] and in predialysis patients with chonic kidney disease [54], an inverse relationship was identified between the levels of DKK1 and presence of plaque formation in patients with primary SS. On the other hand, BMD was positively associated with serum sclerostin, as already suggested, as well as with DKK1 levels, an observation not previously reported [14]. A plausible scenario for the current observations could be that osteoporotic/osteopenic SS patients, characterized by suppressed Wnt signaling, display paradoxically reduced DKK1 levels possibly due to the absence of a negative feedback provided by Wnt inhibitors as previously suggested [14]. As a result, a lack of DKK1mediated protection against vascular wall calcification could lead to plaque formation and increased atherosclerotic risk.

\section{Conclusion}

In conclusion, in the present study we demonstrated that almost two thirds of patients with primary SS have evidence of subclinical atherosclerosis and impaired bone health, which may be attributed in part to the presence of traditional risk factors as well as disease-related features, with Wnt pathway mediators possibly accounting for this observation. Further larger studies are warranted to better explore the contribution of the Wnt signaling pathway in the pathogenesis of these comorbidities in both healthy and autoimmune populations.

\section{Additional files}

Additional file 1: Table S1. Characteristics of primary SS and rheumatoid arthritis (RA) patients and age-sex matched healthy individuals. Table S2. Traditional and disease-related predictors of arterial wall thickening in patients with RA. Table S3. Traditional and disease-related predictors of plaque formation in patients with RA. Table S4. Determinants of osteoporosis or osteopenia in RA patients.

Additional file 2: Figure S1. Increased rates of osteoporosis/osteopenia in patients with primary Sjogren's syndrome (SS) with urine $\mathrm{pH}>5.5$.

\section{Abbreviations}

ANA: antinuclear antibodies; BMD: bone mineral density; BMI: body mass index; CRP: C-reactive protein; CTX: type 1 cross-linked c-telopeptide;

CVD: cardiovascular disease; DKK1: Dickkopf-related protein 1; DM: ciabetes mellitus; ELISA: enzyme-linked immunosorbent assay; ESR: erythrocyte sedimentation rate; ESSDAl: Sjogren's syndrome disease activity index; $\mathrm{HC}$ : healthy controls; HDL: high-density lipoprotein; HT: hypertension; IFN: Interferon; IL-17: Interleukin-17; IMT: intima media thickness; LDL: low-density lipoprotein; OH: hydroxy; OP: osteoporosis; OR: odds ratio; PMH: past medical history; PTH: parathyroid hormone; RA: rheumatoid arthritis; RF: rheumatoid factor; SLE: systemic lupus erythematosous; SS: Sjogren's syndrome; SSA: antibodies to Ro; SSB: antibodies to La; WHO: World Health Organization; Wnt: Wingless-type.

\section{Competing interests}

The authors declare that they have no competing interests.

\section{Authors' contributions}

FG and IP recorded all clinical and laboratory data from all study participants and drafted the manuscript. EA performed all ultrasonographic measurements and revised the manuscript. AN performed ELISA experiments (determination of DKK1 and sclerostin levels) and drafted the manuscript. KM performed determination of autoantibodies and drafted the manuscript. DI and MK supervised patients/sera recruitment, participated in the design and coordination of the study and revised the manuscript. HMM provided SS patients, contributed to study conception, design and data analysis and revised the manuscript. CPM conceived, designed and coordinated the study, performed the statistical analysis and revised the manuscript. All authors read and approved the final manuscript.

\section{Acknowledgements}

The authors would like to acknowledge Hellenic Rheumatology Association and Stavros Niarchos Foundation for financial support and Professor AG Tzioufas, MD, for fruitful suggestions.

\section{Author details}

'Department of Rheumatology, General Hospital of Athens "G.Gennimatas", Athens, Greece. ${ }^{2}$ Department of Pathophysiology, School of Medicine, University of Athens, Athens, Greece. ${ }^{3}$ Department of Radiology, General Hospital of Athens "G.Gennimatas", Athens, Greece. "Department of Physiology, School of Medicine, University of Athens, M. Asias 75, Athens 11527, Greece. ${ }^{5}$ Department of Immunology, General Hospital of Athens "G. Gennimatas", Athens, Greece.

Received: 3 February 2015 Accepted: 30 March 2015

Published online: 11 April 2015

\section{References}

1. Svenungsson E, Jensen-Urstad K, Heimburger M, Silveira A, Hamsten A, de Faire $U$, et al. Risk factors for cardiovascular disease in systemic lupus erythematosus. Circulation. 2001;104:1887-93. 
2. Toloza SM, Uribe AG, McGwin Jr G, Alarcon GS, Fessler BJ, Bastian HM, et al Systemic lupus erythematosus in a multiethnic US cohort (LUMINA). XXIII. Baseline predictors of vascular events. Arthritis Rheum. 2004;50:3947-57.

3. Shoenfeld Y, Gerli R, Doria A, Matsuura E, Cerinic MM, Ronda N, et al. Accelerated atherosclerosis in autoimmune rheumatic diseases. Circulation. 2005;112:3337-47.

4. Giles JT, Post WS, Blumenthal RS, Polak J, Petri M, Gelber AC, et al. Longitudinal predictors of progression of carotid atherosclerosis in rheumatoid arthritis. Arthritis Rheum. 2011;63:3216-25.

5. Meune C, Touze E, Trinquart L, Allanore Y. Trends in cardiovascular mortality in patients with rheumatoid arthritis over 50 years: a systematic review and meta-analysis of cohort studies. Rheumatology (Oxford). 2009;48:1309-13.

6. Salmon JE, Roman MJ. Subclinical atherosclerosis in rheumatoid arthritis and systemic lupus erythematosus. Am J Med. 2008;121:S3-8.

7. Chatterjee Adhikari M, Guin A, Chakraborty S, Sinhamahapatra P, Ghosh A. Subclinical atherosclerosis and endothelial dysfunction in patients with early rheumatoid arthritis as evidenced by measurement of carotid intima-media thickness and flow-mediated vasodilatation: an observational study. Semin Arthritis Rheum. 2012:41:669-75.

8. den Uyl D, Nurmohamed MT, van Tuyl LH, Raterman HG, Lems WF. (Sub)clinical cardiovascular disease is associated with increased bone loss and fracture risk; a systematic review of the association between cardiovascular disease and osteoporosis. Arthritis Res Ther. 2011;13:R5.

9. Zhu TY, Griffith JF, Au SK, Tang XL, Kwok AW, Leung PC, et al. Bone mineral density change in systemic lupus erythematosus: a 5-year followup study. J Rheumatol. 2014;41:1990-7.

10. Lodder MC, Haugeberg G, Lems WF, Uhlig T, Orstavik RE, Kostense PJ, et al. Radiographic damage associated with low bone mineral density and vertebral deformities in rheumatoid arthritis: the Oslo-Truro-Amsterdam (OSTRA) collaborative study. Arthritis Rheum. 2003:49:209-15.

11. Avouac J, Koumakis E, Toth E, Meunier M, Maury E, Kahan A, et al. Increased risk of osteoporosis and fracture in women with systemic sclerosis: a comparative study with rheumatoid arthritis. Arthritis Care Res (Hoboken). 2012;64:1871-8.

12. Mavragani CP, Moutsopoulos HM. The geoepidemiology of Sjogren's syndrome. Autoimmun Rev. 2010;9:A305-310.

13. Yao Q, Altman RD, Wang X. Systemic lupus erythematosus with Sjogren syndrome compared to systemic lupus erythematosus alone: a meta-analysis. J Clin Rheumatol. 2012;18:28-32.

14. Hampson G, Edwards S, Conroy S, Blake GM, Fogelman I, Frost ML. The relationship between inhibitors of the Wnt signalling pathway (Dickkopf 1 (DKK1) and sclerostin), bone mineral density, vascular calcification and arterial stiffness in post-menopausal women. Bone. 2013:56:42-7.

15. Towler DA, Shao JS, Cheng SL, Pingsterhaus JM, Loewy AP. Osteogenic regulation of vascular calcification. Ann NY Acad Sci. 2006;1068:327-33.

16. Vitali C, Bombardieri S, Jonsson R, Moutsopoulos HM, Alexander EL, Carsons $\mathrm{SE}$, et al. Classification criteria for Sjogren's syndrome: a revised version of the European criteria proposed by the American-European Consensus Group. Ann Rheum Dis. 2002;61:554-8.

17. Seror R, Ravaud P, Bowman SJ, Baron G, Tzioufas A, Theander E, et al. EULAR Sjogren's syndrome disease activity index: development of a consensus systemic disease activity index for primary Sjogren's syndrome. Ann Rheum Dis. 2010;69:1103-9.

18. Vaudo G, Schillaci G, Evangelista F, Pasqualini L, Verdecchia P, Mannarino E. Arterial wall thickening at different sites and its association with left ventricular hypertrophy in newly diagnosed essential hypertension. Am J Hypertens. 2000;13:324-31.

19. Vaudo G, Bocci EB, Shoenfeld $Y$, Schillaci G, Wu R, Del Papa N, et al. Precocious intima-media thickening in patients with primary Sjogren's syndrome. Arthritis Rheum. 2005:52:3890-7.

20. Stergiou GS, Salgami EV. New European, American and International guidelines for hypertension management: agreement and disagreement Expert Rev Cardiovasc Ther. 2004;2:359-68

21. 2003 European Society of Hypertension-European Society of Cardiology guidelines for the management of arterial hypertension. J Hypertens. 2003; 21:1011-53

22. Kanis JA, Burlet N, Cooper C, Delmas PD, Reginster JY, Borgstrom F, et al. European guidance for the diagnosis and management of osteoporosis in postmenopausal women. Osteoporos Int. 2008;19:399-428.

23. Assessment of fracture risk and its application to screening for postmenopausal osteoporosis. Report of a WHO Study Group. World Health Organ Tech Rep Ser. 1994; 843:1-129.
24. Tanko LB, Christiansen C, Cox DA, Geiger MJ, McNabb MA, Cummings SR. Relationship between osteoporosis and cardiovascular disease in postmenopausal women. J Bone Miner Res. 2005;20:1912-20.

25. Hyder JA, Allison MA, Wong N, Papa A, Lang TF, Sirlin C, et al. Association of coronary artery and aortic calcium with lumbar bone density: the MESA Abdominal Aortic Calcium Study. Am J Epidemiol. 2009:169:186-94.

26. Full LE, Ruisanchez C, Monaco C. The inextricable link between atherosclerosis and prototypical inflammatory diseases rheumatoid arthritis and systemic lupus erythematosus. Arthritis Res Ther. 2009;11:217.

27. Sen M, Ghosh G. Transcriptional outcome of Wnt-Frizzled signal transduction in inflammation: evolving concepts. J Immunol. 2008;181:4441-5.

28. Kassi E, Adamopoulos C, Basdra EK, Papavassiliou AG. Role of vitamin D in atherosclerosis. Circulation. 2013;128:2517-31.

29. Perez-De-Lis M, Akasbi M, Siso A, Diez-Cascon P, Brito-Zeron P, Diaz-Lagares C, et al. Cardiovascular risk factors in primary Sjogren's syndrome: a case-control study in 624 patients. Lupus. 2010;19:941-8.

30. Juarez M, Toms TE, de Pablo P, Mitchell S, Bowman S, Nightingale P, et al. Cardiovascular risk factors in women with primary Sjogren's syndrome: United Kingdom primary Sjogren's syndrome registry results. Arthritis Care Res. 2014;66:757-64.

31. Cruz W, Fialho S, Morato E, Castro G, Zimmermann A, Ribeiro G, et al. Is there a link between inflammation and abnormal lipoprotein profile in Sjogren's syndrome? Joint Bone Spine. 2010;77:229-31.

32. Bartoloni E, Baldini C, Schillaci G, Quartuccio L, Priori R, Carubbi F, et al. Cardiovascular disease risk burden in primary Sjogren's syndrome: results of a population-based multicentre cohort study. J Int Med. 2015 doi:10.1111/joim.12346.

33. Lodde BM, Sankar V, Kok MR, Leakan RA, Tak PP, Pillemer SR. Serum lipid levels in Sjogren's syndrome. Rheumatology (Oxford). 2006;45:481-4.

34. Roman MJ, Shanker BA, Davis A, Lockshin MD, Sammaritano L, Simantov R, et al. Prevalence and correlates of accelerated atherosclerosis in systemic lupus erythematosus. N Engl J Med. 2003;349:2399-406.

35. Im CH, Kim NR, Kang JW, Kim JH, Kang JY, Bae GB, et al. Inflammatory burden interacts with conventional cardiovascular risk factors for carotid plaque formation in rheumatoid arthritis. Rheumatology (Oxford, England). pii: keu376. Pubmed Epub 2014 Oct 9.

36. Dave AJ, Fiorentino D, Lingala B, Krishnan E, Chung L. Atherosclerotic cardiovascular disease in hospitalized patients with systemic sclerosis: higher mortality than patients with lupus and rheumatoid arthritis. Arthritis Care Res (Hoboken). 2014;66:323-7.

37. Rachapalli SM, Kiely PD, Bourke BE. Prevalence of abnormal ankle brachial index in patients with primary Sjogren's syndrome. Clin Rheumatol. 2009;28:587-90.

38. Gerli R, Vaudo G, Bocci EB, Schillaci G, Alunno A, Luccioli F, et al. Functional impairment of the arterial wall in primary Sjogren's syndrome: combined action of immunologic and inflammatory factors. Arthritis Care Res (Hoboken). 2010;62:712-18.

39. Atzeni F, Sarzi-Puttini P, Signorello MC, Gianturco L, Stella D, Boccassini L, et al New parameters for identifying subclinical atherosclerosis in patients with primary Sjogren's syndrome: a pilot study. Clin Exp Rheumatol. 2014;32:361-8.

40. Kaplan MJ, Salmon JE. How does interferon-alpha insult the vasculature? Let me count the ways. Arthritis Rheum. 2011;63:334-6.

41. Oryoji K, Kiyohara C, Horiuchi T, Tsukamoto H, Niiro H, Shimoda T, et al. Reduced carotid intima-media thickness in systemic lupus erythematosus patients treated with cyclosporine A. Mod Rheumatol. 2014;24:86-92.

42. del Rincon I, Battafarano DF, Restrepo JF, Erikson JM, Escalante A. Glucocorticoid dose thresholds associated with all-cause and cardiovascular mortality in rheumatoid arthritis. Arthritis Rheumatol. 2014;66:264-72.

43. Ghazi M, Kolta S, Briot K, Fechtenbaum J, Paternotte S, Roux C. Prevalence of vertebral fractures in patients with rheumatoid arthritis: revisiting the role of glucocorticoids. Osteoporos Int. 2012;23:581-7.

44. Selmi C, Meroni PL, Gershwin ME. Primary biliary cirrhosis and Sjogren's syndrome: Autoimmune epithelitis. J Autoimmun. 2012;39:34-42.

45. Goules A, Masouridi S, Tzioufas AG, loannidis JP, Skopouli FN, Moutsopoulos HM. Clinically significant and biopsy-documented renal involvement in primary Sjogren syndrome. Medicine (Baltimore). 2000;79:241-9.

46. Moutsopoulos HM, Cledes J, Skopouli FN, Elisaf M, Youinou P. Nephrocalcinosis in Sjogren's syndrome: a late sequela of renal tubular acidosis. J Intern Med. 1991;230:187-91.

47. Jung SM, Kim KW, Yang CW, Park SH, Ju JH. Cytokine-mediated bone destruction in rheumatoid arthritis. Int J Immunol Res. 2014;2014:263625. 
48. Hauser B, Riches PL, Wilson JF, Horne AE, Ralston SH. Prevalence and clinical prediction of osteoporosis in a contemporary cohort of patients with rheumatoid arthritis. Rheumatology (Oxford). 2014;53:1759-66.

49. Zhou R, Zhou H, Cui M, Chen L, Xu J. The association between aortic calcification and fracture risk in postmenopausal women in China: the prospective Chongqing osteoporosis study. PLoS One. 2014;9, e93882.

50. Schulz E, Arfai K, Liu X, Sayre J, Gilsanz V. Aortic calcification and the risk of osteoporosis and fractures. J Clin Endocrinol Metab. 2004:89:4246-53.

51. Claes KJ, Viaene L, Heye S, Meijers B, d'Haese P, Evenepoel P. Sclerostin: Another vascular calcification inhibitor? J Clin Endocrinol Metab. 2013;98:3221-8

52. Register TC, Hruska KA, Divers J, Bowden DW, Palmer ND, Carr JJ, et al Plasma Dickkopf1 (DKK1) concentrations negatively associate with atherosclerotic calcified plaque in African-Americans with type 2 diabetes. J Clin Endocrinol Metab. 2013;98:E60-5.

53. Rachner TD, Khosla S, Hofbauer LC. Osteoporosis: now and the future. Lancet. 2011:377:1276-87.

54. Thambiah S, Roplekar R, Manghat P, Fogelman I, Fraser WD, Goldsmith D, et al. Circulating sclerostin and Dickkopf-1 (DKK1) in predialysis chronic kidney disease (CKD): relationship with bone density and arterial stiffness. Calcif Tissue Int. 2012;90:473-80.

\section{Submit your next manuscript to BioMed Central and take full advantage of:}

- Convenient online submission

- Thorough peer review

- No space constraints or color figure charges

- Immediate publication on acceptance

- Inclusion in PubMed, CAS, Scopus and Google Scholar

- Research which is freely available for redistribution 